\title{
Hyponatremia during a Severe Pneumococcal Meningitis with Renal Salt Wasting: A Cerebral Salt Wasting Syndrome?
}

\author{
—Diagnosing a Cerebral Salt Wasting Syndrome
}

\author{
Jean-Philippe Delabre, Stephane Pommet, Laurent Amigues, Olivier Jonquet, Kada Klouche \\ Department of Intensive Care Unit Lapeyronie University Hospital, Montpellier, France \\ E-mail: k-klouche@chu-montpellier.fr \\ Received September 11, 2011; revised September 19, 2011; accepted September 26, 2011
}

\begin{abstract}
Background: The existence and prevalence of cerebral salt wasting and its differentiation from syndrome of inappropriate antidiuretic hormone have been controversial. This controversy stems from overlapping clinical and laboratory findings and an inability to assess the volume status of these patients. Objectives:To present a case of a cerebral salt wasting syndrome secondary to a bacterial mengitis and to emphasize the difficulty to assess the diagnosis. Case report: A 51-year-old male admitted to the ICU for a severe bacterial meningitis who developed, four days later, hyponatremia associated with hypovolemia due to a renal salt wasting. Clinical and biological parameters were collected; electrolytes balances and salt clearances were calculated to best investigate this hydroelectrolytic disorder. Patient's volume status and improvement after fluid and sodium replacement highly suggests the diagnosis of a cerebral salt wasting syndrome. Conclusion: A thorough analysis of this case showed that occurrence of hyponatremia and renal salt wasting after brain agression is not univocal underlying the fact that the diagnosis of cerebral salt wasting should be assessed only after a careful examination of the different determinants of the hydroelectrolytical balance.
\end{abstract}

Keywords: Bacterial Meningitis, Cerebral Salt Wasting, Hyponatremia, Natriuresis

\section{Introduction}

Hyponatremia is a common electrolyte disturbance in patients after central nervous system disease [1,2]. The syndrome of inappropriate secretion of antidiuretic hormone (SIADH) is the main attributed cause to this disorder [3]. In some few cases, however, hyponatremia may be due to a renal salt wasting and diagnosed as a cerebral salt wasting syndrome (CSWS). CSWS, first described by Peters in 1950, is defined as the occurrence of an elevated diuresis and natriuresis after a cerebral injury, leading to hyponatremia and hypovolemia [4]. Despite substantial evidence in animal experiments, its pathophysiology and pertinency remains a question of debate in clinical settings [5]. We report herein the case of a patient who developped hyponatremia with renal salt wasting associated with hypovolemia following a severe bacterial meningitis suggestive of CSWS. Since many different mechanisms can lead to hyponatremia and a diagnosis of CSWS is seldom univocal, we thoroughly analyzed electrolytes balances, and salt clearances to assess the diagnosis.

\section{Case Report}

A 51 year old male was admitted in our ICU with fever, headache and drowsiness evolving since 24 hours. At admission, the computed tomography (CT) scann was normal. The lumbar puncture was purulent with 7000 leucocytes per $\mathrm{mm}^{3}, 95 \%$ being polynuclears. Cefotaxime and vancomycin in association with dexamethasone were administered to the patient. At day 1 , he required mechanical ventilation because its consciousness deteriorated. Cerebrospinal fluid was positive with penicillinsensitive streptococcus pneumoniae and antibiotherapy was switched to amoxicillin. At day 5, the patient significantly improved and was weaned of mechanical ventilation with a good neurological status. 
From day 4, he developed a polyuria with daily diuresis between 4.4 and 5.7 liters which persisted four days leading to a weight loss of $6.5 \mathrm{~kg}$, clinical evidence of hypovolemia, and an increase of blood urea nitrogen (BUN) and hematocrit. In the same time, natremia decreased from 145 to $126 \mathrm{mmol} / \mathrm{l}$ and natriuresis increased from 15 to $820 \mathrm{mmol} /$ day. Clinico-biological disorders are summarized on Tables 1 and 2. Cortisolemia was normal at $8 \mathrm{am}$, phosphore excretion fraction was 18\% and chromatography of urinary aminoacids was normal. Dehydration with inappropriate natriuresis evoked the occurrence of a renal salt wasting. The association of this tubulopathy with the cerebral agression was highly suggestive of the diagnosis of CSWS. Rehydratation and salt supplementation enabled, as shown in Table 2, correction of hypovolemia and hyponatremia, which increased to $138 \mathrm{mmol} / \mathrm{l}$ at day 10 .

\section{Discussion}

Hyponatremia is common in the setting of central nervous system disease and is associated with significant morbidity and mortality rates [6]. One third of patients in the acute phase of subarachnoid haemorrhage and more than one third of neurosurgical patients especially following transsphenoidal surgery suffer from this electrolyte disorder [6,7]. It is often attributed to SIADH. When hyponatremia with increased urine $\mathrm{Na}^{+}$concentration is associated with a contracted extracellular fluid volume, it may however be caused by renal salt wasting resulting from a centrally mediated process and diagnosed as a CSWS. The diagnosis of a CSWS requires the presence of hyponatremia with hypovolemia associated with an elevated natriuresis (sodium clearance $>200 \mathrm{ml} /$ day) after a cerebral injury in the exclusion of the current causes of renal salt wasting: diuretics use, Bartter and Gitelman syndrome, hypercalemia, renal failure, hypervolemia correction, metabolic alkalosis and corticosteroid insufficiency. Increased secretion of natriuretic peptides like brain natriuretic peptide (BNP) and atrial natriuretic peptide (ANP) may be responsible for the excessive urinary Na excre tion [8]. Disruption of kidney neural input may also leads to renal salt wasting [9]. The precise mechanism of CSWS remains however poorly understood.

Table 1. Biological and clinical data measured at 8:00 am. (MAP: mean arterial pressure, BUN: blood urea nitrogen, [Na]p: plasmatic sodium, Osm plasm : plasmatic osmolality. ND: not determinated).

\begin{tabular}{cccccccccc}
\hline & D1 & D2 & D3 & D4 & D5 & D6 & D7 & D8 & D9 \\
\hline Clinical & & & & & & & & & \\
Weight (kg) & 68 & 70 & 72.2 & 73.5 & 71.5 & 68 & 67 & 66 & 66 \\
MAP (mmHg) & 105 & 100 & 110 & 95 & 105 & 100 & 80 & 100 & 98 \\
Biological & & & & & & & & & \\
BUN (mmol/l) & & 6 & & & & & & \\
Hematocrit (\%) & 135 & 143 & 141 & 145 & 140 & 140 & 126 & 132 & 133 \\
[Na]p (mmol/l) & 70 & 60 & 57 & 60 & 62 & 64 & 77 & 76 & 69 \\
Protidemia (g/l) & & & & & & & 268 & & \\
Osm plasm (mosm/l) & & & & & & & & & \\
\end{tabular}

Table 2. Data measured and calculated on 24 hours period. ([Na]u + [K]u: sum of the kaliuresis and natriuresis concentrations, Dexa: Dexamethasone).

\begin{tabular}{|c|c|c|c|c|c|c|c|c|}
\hline & D1 - D2 & D2 - D3 & D3 - D4 & D4 - D5 & D5 - D6 & D6 - D7 & D7 - D8 & D8 - D9 \\
\hline \multicolumn{9}{|l|}{ Urine } \\
\hline Urine output (ml/d) & 1400 & 1800 & 1250 & 4400 & 4700 & 5300 & 5700 & 3100 \\
\hline$[\mathrm{Na}] \mathrm{u}+[\mathrm{K}] \mathrm{u}(\mathrm{mmol} / \mathrm{d})$ & 26.6 & ND & 176 & 792 & 855 & 1118 & 707 & 484 \\
\hline \multicolumn{9}{|l|}{ Infusions } \\
\hline Infused volume (ml/d) & 5820 & 5000 & 3320 & 3100 & 2050 & 3500 & 4750 & 3227 \\
\hline $\mathrm{Na}+$ and $\mathrm{K}+$ infused (mmol/day) & 726 & 588 & 202 & 146 & 165 & 182 & 290 & 199 \\
\hline Sodium clearance (ml/d) & 110 & ND & 926 & 5002 & 5740 & 6688 & 3901 & 2480 \\
\hline Isonatric balance (ml) & +4388 & ND & +163 & -3976 & -4323 & -6054 & -2760 & -1873 \\
\hline \multirow[t]{2}{*}{ Associated treatments } & Dexa & Dexa & Dexa & furosemide & & & & \\
\hline & Intubation & & & & Extubation & & & \\
\hline
\end{tabular}


CSWS is yet unrecognized and its incidence is variably estimated among authors [1-3,10]. In the study of Sherlock, hyponatremia after subarachnoid haemorrhage was induced by a CSWS in $6.5 \%$ of cases and by SIADH in $69 \%$ of cases [10]. A few observations of CSWS after infectious meningitis have been published and they were mainly tuberculous or viral origin [11-13]. Yet, at the best of our knowledge, only two cases of bacterial meningitis with a CSWS has been reported [14,15]. This report adds a new observation of a CSWS after bacterial meningitis, but also underlines the difficulty to assess this diagnosis particularly in ICU settings. Indeed, our patient developed at day 7 of a severe pneumococcal meningitis a clinical disorder associating dehydration, hyponatremia and renal salt wasting. Significant weight loss, increase of BUN and hematocrit support the fact that he was hypovolemic. Moreover, the occurrence of polyuria and correction of the disorder by saline infusion ruled out the diagnosis of SIADH. Main etiologies of tubulopathy have been also ruled out (calcemia, kaliemia, acido-basic status, cortisolemia and renal function were normals) but multiple factors that may play a role must be taken into account to secure the diagnosis. Firstly, the patient exhibited three days before occurrence of hyponatremia (at day 4), an opposite situation associating hypernatremia and hypervolemia as a result of a net gain of isonatric volume (infusion of isotonic saline) and the occurrence of a low clearance of sodium (Table 2). Decreased urinary Na excretion was secondary to the association of corticotherapy and positive pressure ventilation. Secondarly, natriuresis and diuresis increased after day 4 leading to an increase of Na clearance and a dramatic decrease of isonatric balance to day 8 as displayed in Table 2. This results probably from the occurrence of a CSWS but also, for a part, from positive pression ventilation interruption, and discontinuation of dexamethasone (Table 2). Unless integrating all these elements, it seems therefore somewhat difficult to assess definitively the diagnosis of CSWS and this should be a general rule in hyponatremia diagnosis in similar circumstances. Nonetheless, rehydration and salt loading, that is therapeutic management of CSWS, best corrected the disorder.

\section{Conclusions}

The case of our patient is an illustration of the problem in diagnosing a CSWS: the appearance of a renal salt wasting syndrome with hypovolemia and hyponatremia after a cerebral injury is insufficient to claim the presence of a CSWS. In fact, such patients generally benefit from several treatments affecting the hydroelectrolytical balance: isotonic saline, positive pressure ventilation, corticoste- roids and mannitol. Even after excluding major causes of tubulopathy, the presence of a CSWS should be assessed only after a careful examination of the different determinants of the hydroelectrolytical balance, and considering cessation of treatments.

\section{References}

[1] M. Cerda-Esteve, A. Ruiz-González, M. Gudelis, A. Goday, J. Trujillano and E. Cuadrado, et al., "Incidence of Hyponatremia and Its Causes in Neurological Patients," Endocrinol Nutrition, Vol. 5, No. 57, 2010, pp.182-186. doi:10.1016/j.endonu.2010.02.009

[2] M. G. Betjes, "Hyponatremia in Acute Brain Disease: The Cerebral Salt Wasting Syndrome,” European Journal of Internal Medicine, Vol. 1, No 13, 2002, pp. 9-14. doi:10.1016/S0953-6205(01)00192-3

[3] N. Moro, Y. Katayama, T. Igarashi, T. Mori, T. Kawamata and J. Kojima, "Hyponatremia in Patients with Traumatic Brain Injury: Incidence, Mechanism, and Response to Sodium Supplementation or Retention Therapy with Hydrocortisone,” Surgery Neurological, Vol. 4, No. 68, 2007, pp. 387-393. doi:10.1016/j.surneu.2006.11.052

[4] J. P. Peters, G. Weltg, E. A. H. Sims, J. Orloff and J. Needham, "A Salt-Wasting Syndrome Associated with Cerebral Disease," Transactions of the Association of American Physicians, No. 63, 1950, pp. 57-64.

[5] J. Kojima, Y. Katayama, N. Moro, H. Kawai, M. Yoneko and T. Mori, "Cerebral Salt Wasting in Subarachnoid Hemorrhage Rats: Model, Mechanism, and Tool," Life Science, Vol. 20, No. 76, 2005, pp. 2361-2370.

[6] B. Zheng, Y. Qiu, H. Jin, L. Wang, X. Chen and C. Shi, et al., "A Predictive Value of Hyponatremia for Poor Outcome and Cerebral Infarction in High-Grade Aneurysmal Subarachnoid Haemorrhage Patients," Journal of Neurol Neurosurg Psychiatry, Vol. 2, No. 82, 2011, pp. 213-217. doi:10.1136/jnnp.2009.180349

[7] R. A. Kristof, M. Rother, G. Neuloh and D. Klingmüller, "Incidence, Clinical Manifestations, and Course of Water and Electrolyte Metabolism Disturbances Following Transsphenoidal Pituitary Adenoma Surgery: A Prospective Observational Study," Journal of Neurosurg, Vol. 3, No. 111, 2009, pp. 555-562. doi:10.3171/2008.9.JNS08191

[8] E. Berendes, M. Walter, P. Cullen, T. Prien, H. Van Aken and J. Horsthemke, et al., "Secretion of Brain Natriuretic Peptide in Patients with Aneurysmal Subarachnoid Haemorrhage," Lancet, Vol. 349, No. 9047, 1997, pp. 245-249. doi:10.1016/S0140-6736(96)08093-2

[9] B. F. Palmer, "Hyponatremia in Patients with Central Nervous System Disease: SIADH Versus CSW,” Trends Endocrinol Metab, Vol. 4, No. 14, 2003, pp. 182-187. doi:10.1016/S1043-2760(03)00048-1

[10] M. Sherlock, E. O’Sullivan, A. Agha, L. A. Behan, D. Rawluk and P. Brennan, et al., "The Incidence and Pathophysiology of Hyponatraemia After Subarachnoid Haem- 
orrhage," Clinical Endocrinol, Vol. 3, No. 64, 2006, pp. 250-254. doi:10.1111/j.1365-2265.2006.02432.x

[11] L. K. Ti, S. C. Kang, and K. F. Cheong, “Acute Hyponatraemia Secondary to Cerebral Salt-Wasting Syndrome in a Patient with Tuberculous Meningitis," Anaesth Intensive Care, Vol. 4, No.26, 1998, pp. 420-423.

[12] U. S. Celik, D. Alabaz, D. Yildizdas, E. Alhan, E. Kocabas and S. Ulutan, "Cerebral Salt-Wasting in Tuberculous Meningitis: Treatment with Fludrocortisone," Ann Trop Paediatr, Vol. 4, No. 25, 2005, pp. 297-302. doi:10.1179/146532805X72458

[13] M. J. Brookes and T. H. Gould, "Cerebral Salt-Wasting
Syndrome in Meningoencephalitis: A Case Report," Journal of Neurol Neurosurg Psychiatry, Vol. 2, No. 74, 2003, pp. 277. doi:10.1136/jnnp.74.2.277

[14] H. Attout, S. Guez and C. Seriès, "Cerebral Salt-Wasting Syndrome in Bacterial Meningitis," Ann Endocrinol, Vol. 5, No. 68, 2007, pp. 395-397.

[15] M. H. Benito, P. Iglesias, P. Guevara, R. Sánchez Hernán-Dez and M. J. Fernández-Reyes, "Hyponatremia Secondary to Cerebral Salt-Wasting Syndrome Associated to Bacterial Meningitis,” Nefrologia, Vol. 5, No. 28, 2008, pp. 570-571. 